\title{
Classifications Used in Total Hip Arthroplasty
}

\author{
Munis Ashraf \\ Additional information is available at the end of the chapter
}

http://dx.doi.org/10.5772/intechopen.77231

\begin{abstract}
Total hip arthroplasty is one of the most successful operation to be done and is definitely a rewarding procedure for both the surgeon and the patient. Ever since 3 days of low friction arthroplasty by Sir John Charnley, there has been considerable interest in improvement in the knowledge of surgical techniques and hip biomechanics. Over the past two decades there has been an exponential increase in total hip replacements. Therefore strategies to simplify the procedure and classifications to encounter difficulties in treatment plans were devised. It is imperative for consultants and trainees to be aware of these classifications systems which are helpful in pre op, intra op and postop planning.
\end{abstract}

Keywords: Vancouver, Paprosky, Brooker

\section{Paprosky classification of acetabular deficiencies for revision hip arthroplasty}

\subsection{Introduction}

Wayne Paprosky (Illinois, USA) proposed this classification in 1994 based on his experience with revision of 134 acetabular cups [1] (Figure 1).

\subsection{Classification}

Type I: Defect with undistorted rim.

Type II: Defect with distorted rim but adequate to support a hemispherical cup.

- IIA: Superior and medial with intact superior rim. 


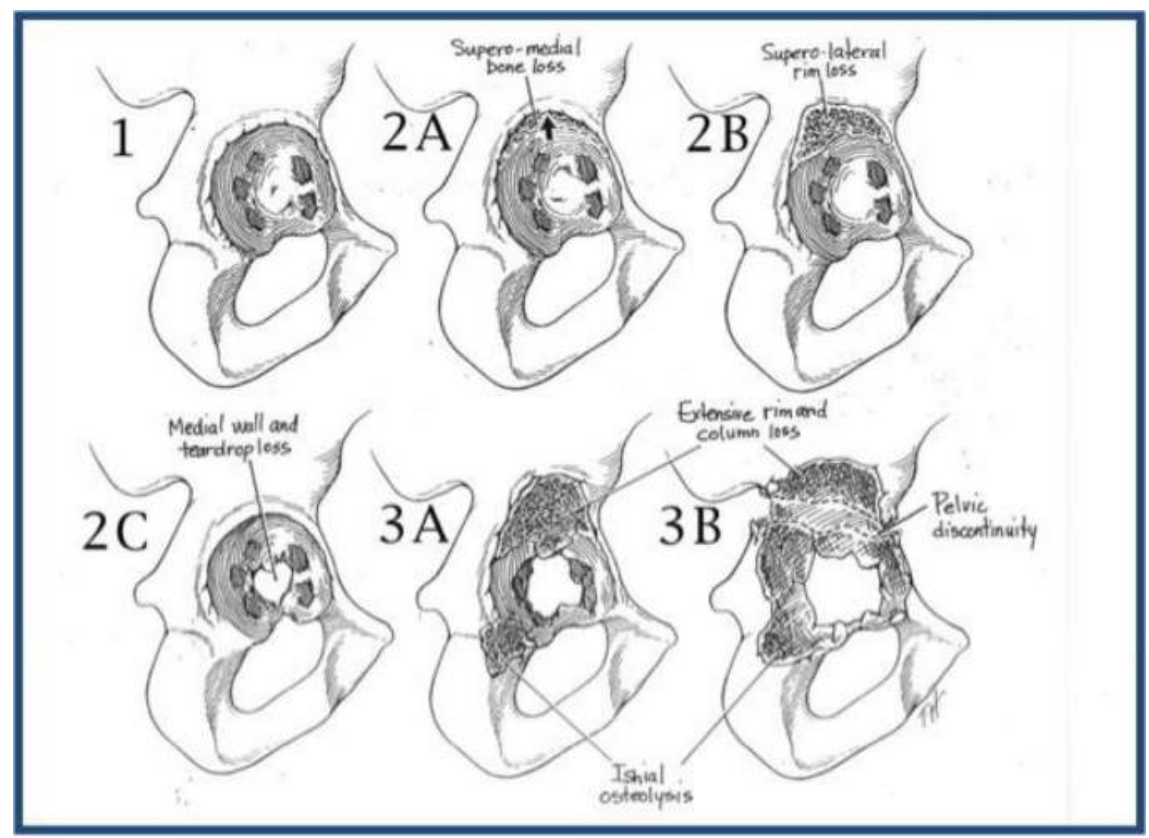

Figure 1. Paprosky classification of acetabular deficiencies.

- IIB: Superior with less than one-third superior rim deficient.

- $\quad$ IIC: Medial wall defect.

Type III: Defect with non-supportive rim.

- III A: Superior and lateral with 40 to $60 \%$ of the host bone intact and partial inherent mechanical stability.

- III B: Superior and medial with host bone less than $40 \%$ and possibility of occult discontinuity.

\subsection{Clinical applications}

Bone grafting techniques depends on the type of acetabular bone defect. Superior dome defect will need structural distal femoral allografts or trabecular metal wedges. Medial wall and ischial defect will need particulate bone grafts. Pelvic discontinuity needs ORIF versus triflanged custom cage. Unsupportive bone stock will need cup and cage construct.

\subsection{Reliability}

Gozzard et al. had performed a study to assess the reliability and validity of classification systems used for defects in acetabulum during revision arthroplasty [2]. It was found that there was poor to good intra observer agreement with the consultants (0.24) and moderate to good intra observer agreement with the registrars (0.36). Interobserver agreement was noted to 
be moderate with consultant and registrars scoring 0.56 and 0.27 , respectively. Validity was deemed to be good $(\kappa=0.65)$. Overall, the authors found the system to be unreliable.

\section{Saleh classification of acetabular deficiencies for revision hip arthroplasty}

\subsection{Introduction}

This system of classification was proposed by Saleh et al. in 1999. The study had included 21 expert arthroplasty surgeons and was proposed based on estimation of anticipated bone stock following implant removal [3] (Figure 2).

\subsection{Classification}

Type I No significant bone loss.

Type II Contained loss of bone stock where there is cavitary enlargement of the acetabular cavity but no wall deficiency.

Type III Uncontained loss of bone stock where there is $<50 \%$ segmental loss of the acetabulum involving anterior or posterior column.

Type IV Uncontained loss of bone stock where there is $>50 \%$ segmental loss of the acetabulum affecting both anterior or posterior columns (if there is $>50 \%$ loss of the acetabulum, involving
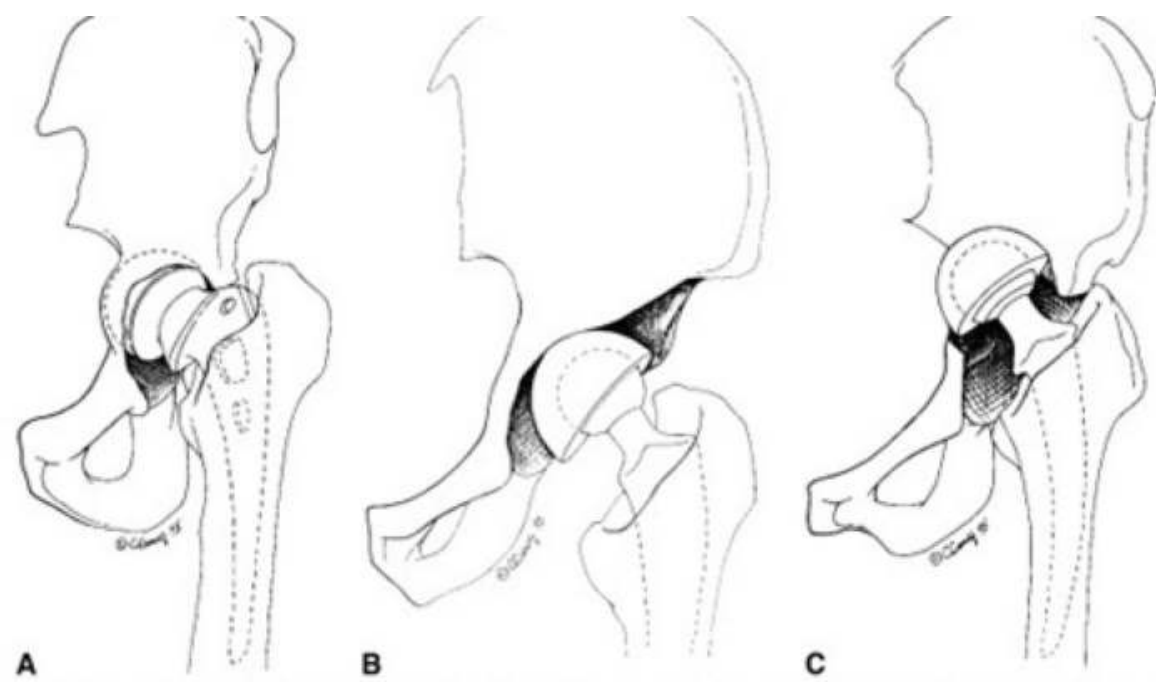

Figure 2. Saleh classification. (A) Type uncontained cavitary loss of bone stock. (B) Type III, uncontained (segmental) loss of bone stock involving $<50 \%$ of acetabulum. (C) Type IV, uncontained (segmental) loss of bone stock involving $>50 \%$ of the acetabulum. 
mostly the medial wall but the columns are intact, then this type of defect is considered type II because of the availability of the columns for reconstruction).

Type V Acetabular defect with uncontained loss of bone stock in association with pelvic discontinuity.

\subsection{Reliability}

Gozzard et al. had observed an Inter-observer reliability testing revealed kappa values of 0.89 for the acetabulum. Average validation value was kappa $=0.86$ for the acetabulum [2]. To put things into perspective: clinical epidemiologists consider correlation values of $0.6-0.8$ to be "substantial" and between 0.8 and 1.0 to be "perfect association".

\section{Hodgkinson classification of radiographic demarcation of the socket following total hip arthroplasty}

\subsection{Introduction}

This classification was proposed by Hodgkinson et al. from Wrightington, UK in 1988. He reviewed 200 patients undergoing revision arthroplasty and found out strong correlation between the extent of radiographic demarcation at bone-cement interface and intraoperative loosening of cemented acetabular components [4].

\subsection{Classification}

Type 0: No demarcation.

Type 1: Demarcation of outer one-third.

Type 2: Demarcation of outer and middle thirds.

Type 3: Complete demarcation.

Type 4: Socket migration.

\subsection{Clinical significance}

This classification helps surgeon help decide between partial or complete revision preoperatively.

\section{Paprosky classification of femoral bone deficiencies}

\subsection{Introduction}

This classification was described by Wayne Paprosky et al. from Illinois, USA. He emphasized that his classification will help the surgeon determine the most appropriate option for 


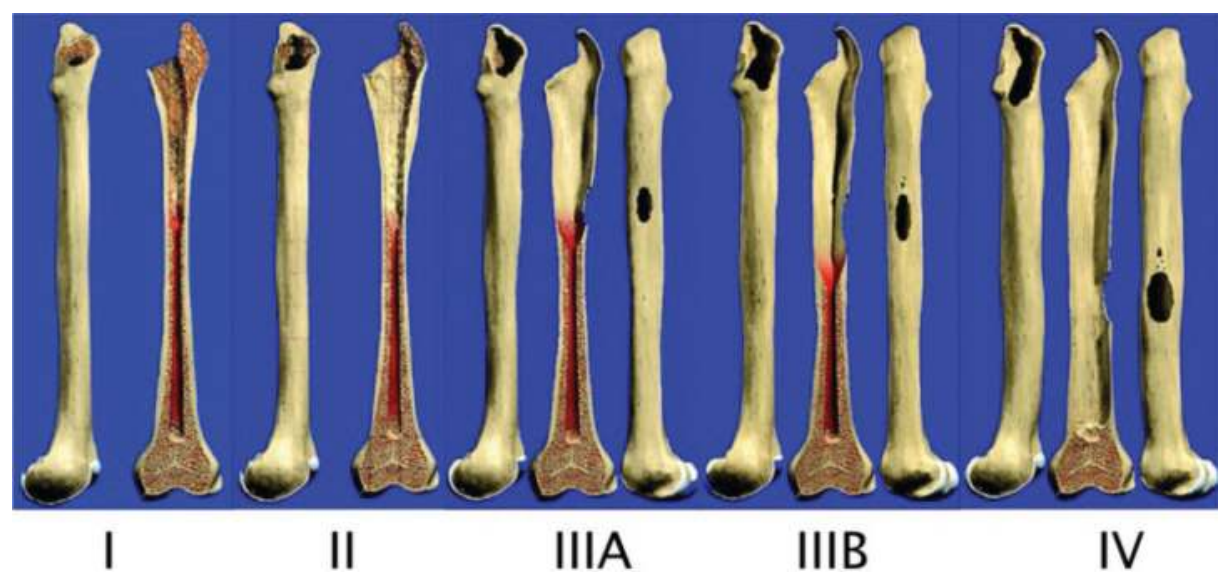

Figure 3. Paprosky classification.

reconstruction and thereby assists with ensuring that the appropriate implants and instruments are available at the time of surgery [5]. Gozzard et al. found moderate agreement between the preoperative and intraoperative validity; but the reliability of the classification was found to be fair (Figure 3).

\subsection{Classification}

Type 1: Minimal metaphyseal and diaphyseal bone loss.

Type 2A: Absent calcar extend just below the inter-trochanteric region.

Type 2B: Anterolateral metaphyseal bone loss wit absent calcar.

Type 2C: Posteromedial metaphyseal bone loss.

Type 3A: 2A plus diaphyseal bone loss but at least $4 \mathrm{~cm}$ of diaphyseal support possible.

Type 3B: 2B plus diaphyseal bone loss with less than $4 \mathrm{~cm}$ of diaphyseal support available.

Type 3C: 2C plus complete diaphyseal bone loss.

\subsection{Clinical applications}

Type 1: Cemented or proximally porous coated cementless implant can be used.

Type 2A, 2B, 2C: Extensively porous coated cementless stem is preferred. Cemented stem should be avoided because of loss of metaphyseal endosteal bone.

Type 3A: Extensively porous coated stems or modular distal fitting tapered stems can be used.

Type 3B: Modular tapered cementless stems are used if adequate bone stock.

Impaction bone grafting is also an option. 
Type 4: Impaction bone grafting with tapered cemented stem if intact cortex. Composite prosthesis allograft if no proximal cortex. Long cemented stem is an option in elderly.

\section{AAOS classification of femoral bone deficiencies for revision hip arthroplasty}

\subsection{Introduction}

This classification was first proposed by D'Antonio et al. (Pennsylvania, USA) in 1989 and later adopted by American Academy of Orthopedic Surgeons (AAOS) [6] (Figure 4).

\subsection{Classification}

Type I: Segmental deficiencies.

1a: Proximal either partial or complete.

1b: Intercalary.

1c: Greater trochanteric.

Type II: Cavitary deficiencies (cancellous, cortical or ectasia).

Type III: Combined.

Type IV: Rotational or angular malalignment.

Type V: Femoral stenosis.

Type VI: Femoral discontinuity.

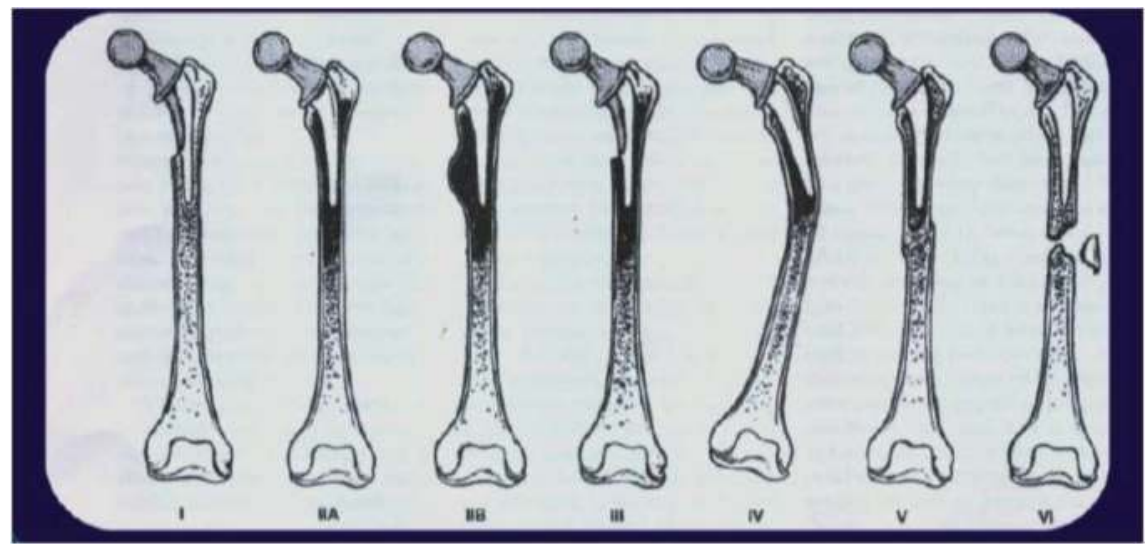

Figure 4. AAOS classification. 


\subsection{Clinical applications}

This classification is very useful in describing the bone defect accurately but has less role in guiding the surgeon determine the reconstructive option.

\subsection{Reliability}

Gozzard et al. in their study observed the inter observer agreement among consultants and registrars. They noted a fair agreement ( $k$ value of 0.28 ) among consultants and a poor agreement ( $\mathrm{k}$ value of -1.0$)$ among the registrars.

\section{Saleh classification of femoral bone deficiencies}

\subsection{Introduction}

This system of classification was proposed by Saleh et al. in 1999. The study had included 21 expert arthroplasty surgeons and was proposed based on estimation of anticipated bone stock following implant removal [3].

\subsection{Classification}

\begin{tabular}{lll}
\hline Type & Defect & Treatment \\
\hline I & No significant loss of bone stock. & $\begin{array}{l}\text { Conventional cemented } \\
\text { Uncemented components }\end{array}$ \\
II $\quad$ Contained loss of bone stock, cortical sleeve intact & $\begin{array}{l}\text { Proximal fixation } \\
\text { Impaction grafting } \\
\text { Porous coated implant } \\
\text { Modular implant }\end{array}$ \\
& $\begin{array}{l}\text { Non-circumferential loss of bone stock uncontained } \\
\text { Proximal circumferential loss of bone stock less than } 5 \mathrm{~cm} \\
\text { in length }\end{array}$ & $\begin{array}{l}\text { Cortical strut allograft } \\
\text { Calcar replacing prosthesis }\end{array}$ \\
IV $\quad \begin{array}{l}\text { Circumferential loss of bone stock more than } 5 \mathrm{~cm} \text { in } \\
\text { length (distal to lesser trochanter) }\end{array}$ & $\begin{array}{l}\text { Custom implant, proximal femoral allograft } \\
\text { Periprosthetic fracture with proximal circumferential loss } \\
\text { of bone stock. }\end{array}$ & $\begin{array}{l}\text { Restoration of bone stock plus long stem femoral } \\
\text { component custom implant proximal femoral allograft. }\end{array}$ \\
\end{tabular}

\subsection{Reliability}

In the study by Saleh et al., they noted an inter observer reliability of $k$ value 0.88 and average validity, $\mathrm{k}$ value of 0.88 ; indicating perfect association. The classification also provides probable treatment options for each type. 


\section{Dossick and Dorr classification of proximal femoral geometry}

\subsection{Introduction}

Based on the calcar-to-canal ratio which is defined as the diameter of the femur at the midportion of the lesser trochanter divided by the diameter at a point $10 \mathrm{~cm}$ distal [7].

\subsection{Classification}

Type A: Calcar-to-canal ratio $<0.5$.

No thinning of cortices on AP or lateral radiographs.

Type B: Calcar-to-canal ratio 0.5-0.75.

Thinning of the posterior cortex on the lateral view.

Type C: Calcar-to-canal ratio $>0.75$.

Thinning of cortices on both views (Stovepipe femur) (Figure 5).

\subsection{Clinical significance}

Type A suitable for cementless femoral stem, type $C$ requires use of cemented stem and type B is intermediate.

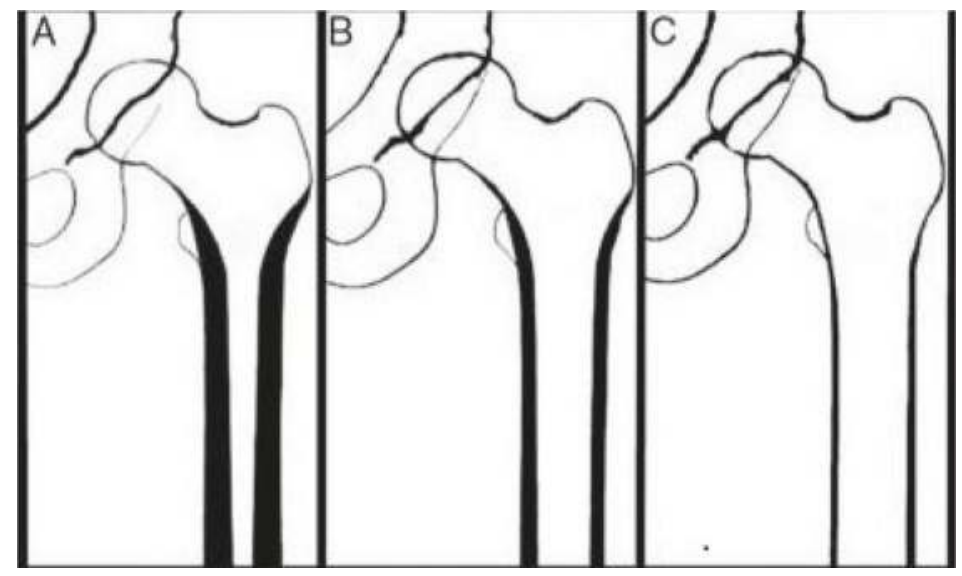

Figure 5. Dossick and Dorr classification. 


\section{Vancouver classification of intraoperative periprosthetic femur fractures around total hip arthroplasty}

\subsection{Classifications}

Type A: Proximal metaphyseal.

A1: Cortical perforation.

A2: Undisplaced linear crack.

A3: Displaced or unstable fractures.

Type B: Proximal diaphyseal.

B1: Cortical perforation.

B2: Undisplaced linear crack.

B3: Displaced or unstable fractures.

Type C: Distal diaphyseal fractures.

C1: Cortical perforation.

C2: Undisplaced linear crack.

C3: Displaced or unstable fracture [8].

\subsection{Clinical applications}

Type A1: Bone graft alone.

Type A2: Circelage wire if using proximally porous coated stem and can be ignored if using fully porous coated stem and there is no distal extension into diaphysis.

Type A3: Needs fixation.

Type B1: Bypassing stem \pm cortical allograft fixation.

Type B2: Circelage wire \pm cortical allograft fixation.

Type B3: Long stem with cortical allograft fixation.

Type C1: Morselized bone graft \pm bypass stem and cortical allograft.

Type C2: Circelage wire \pm bypass stem and cortical allograft.

Type C3: ORIF (Figure 6). 

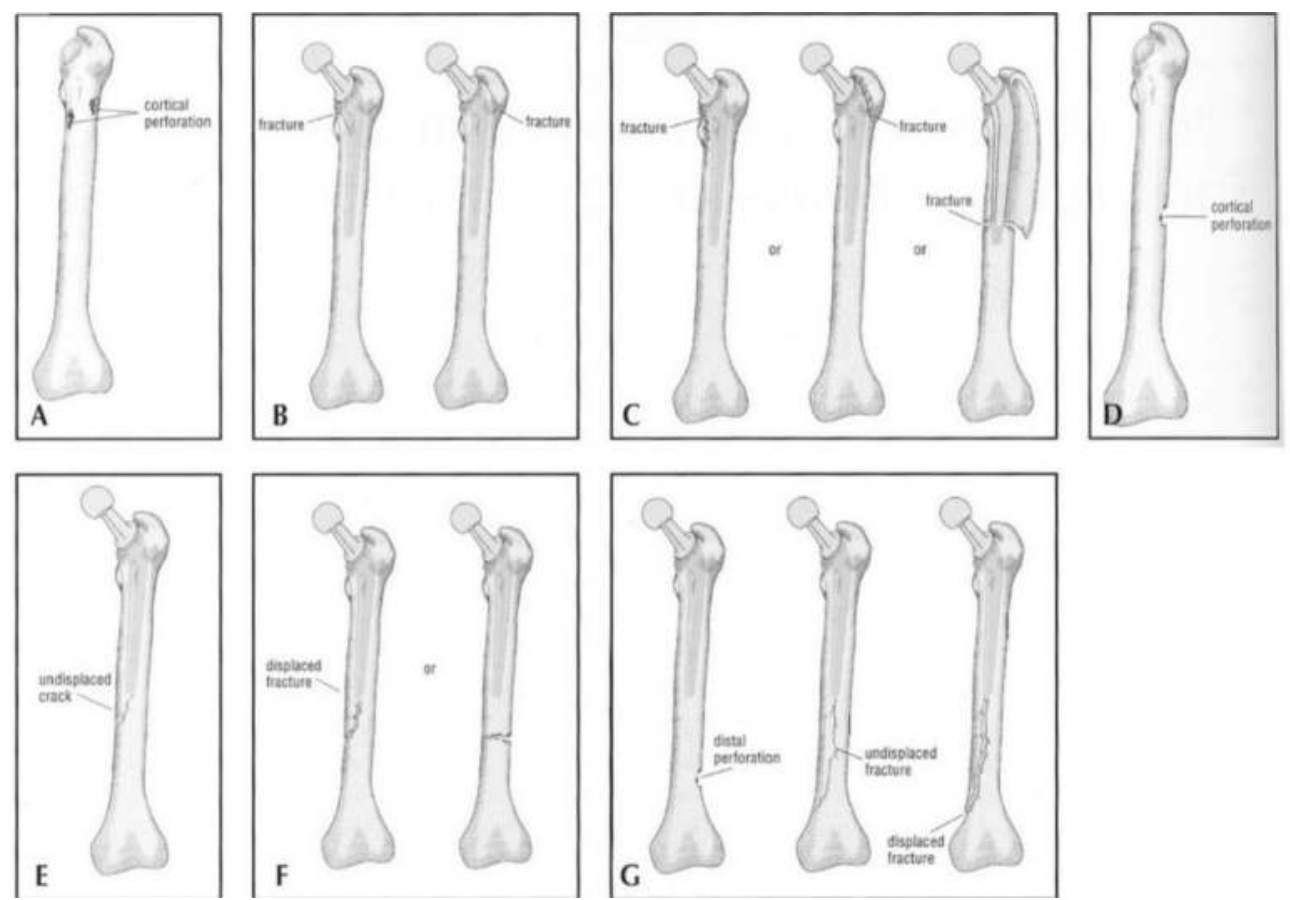

Figure 6. Intraoperative Vancouver classification.

\section{Vancouver classification of postoperative periprosthetic femur fractures} around total hip arthroplasty

\subsection{Classification}

Type A: Peritrochanteric.

AG: Greater trochanter.

AL: Lesser trochanter.

Type B: Around or just distal to the tip of the stem.

B1: Well-fixed femoral component.

B2: Loose femoral component.

B3: Loose femoral component and poor bone stock.

Type C: Well distal to the stem. 


\subsection{Clinical application}

This classification guides the surgeon with treatment decision.

AG and AL: Usually stable and can be treated non-operatively.

B1: ORIF if displaced.

B2: Revision to long stem.

B3: Revision with struct grafting.

C: ORIF.

\subsection{Reliability}

A European validation for this classification was performed by Rayan et al. The study had included consultants, trainees and medical students. It was noted to have an inter observer reliability of substantial agreement among consultants (k value of $0.72-0.74$ ) orthopedic trainees ( $\mathrm{k}$ value of $0.68-0.70$ ) and medical students ( $\mathrm{k}$ value of 0.61 ). The validity within B type fractures revealed an agreement of $77 \%$ with a $\mathrm{k}$ value of $0.67[9,10]$ (Figure 7).
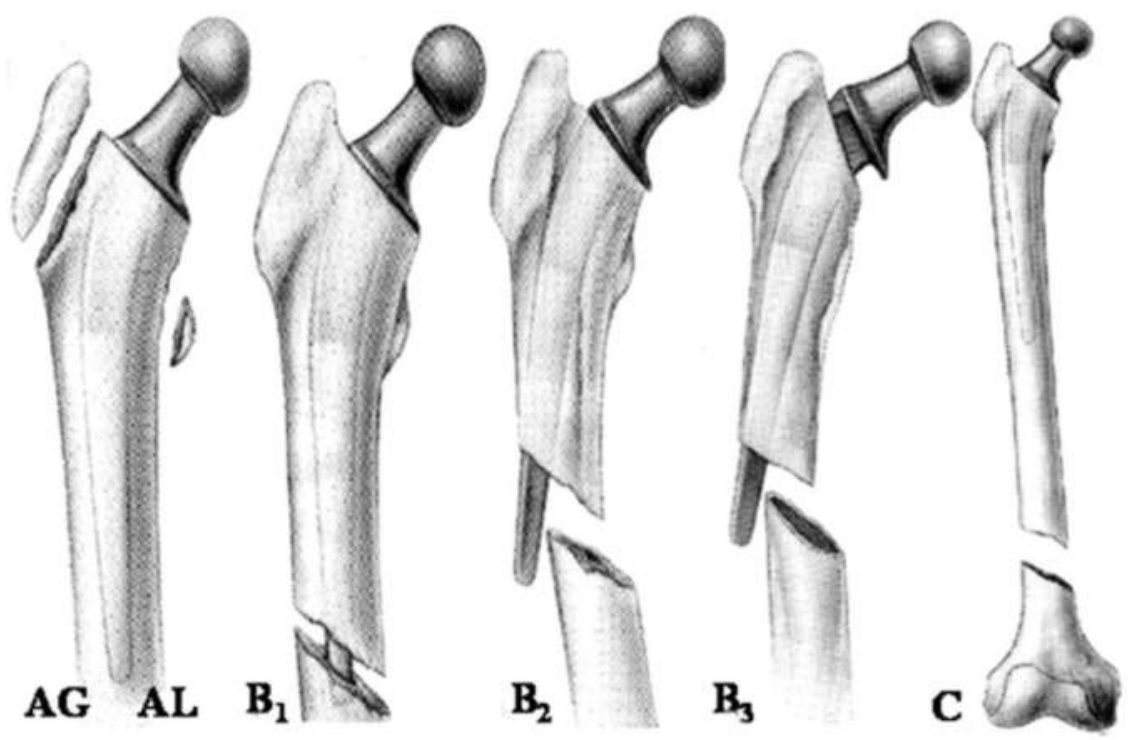

Figure 7. Postoperative Vancouver classification. 


\section{Tsukayama classification of infected hip joint prosthesis}

\subsection{Introduction}

Tsukayama et al. proposed the classification based on the study of 97 patients with infected hip joint prosthesis [11].

\subsection{Classification}

Positive intraoperative cultures: Two out of five intraoperative specimens positive on culture in a patient undergoing revision hip arthroplasty with no clinical evidence of infection at the time of revision.

Early postoperative infection: Wound infection developed less than 1 month after the operation. Late chronic infection: Wound infection developed 1 month or more after the index operation and with insidious course.

Acute hematogenous infection: Associated with a documented or suspected antecedent bacteremia and characterized by an acute onset of symptoms in the affected joint with the prosthesis.

\subsection{Clinical implication (treatment guidelines)}

Positive intraoperative cultures: Intravenous administration of antibiotics for 6 weeks without operative intervention.

Early postoperative infection: Debridement, replacement of the polyethylene inserts of the acetabular component, retention of the prosthesis, and intravenous administration of antibiotics for 4 weeks.

Late chronic infection: Debridement; removal of all prosthetic components and bone cement; and placement of antibiotic beads. Intravenous antibiotics for 6 weeks. Revision arthroplasty 2 weeks after cessation of antibiotic therapy.

Acute hematogenous infection: Debridement, replacement of the polyethylene insert, retention of the prosthesis if it was not loose, and intravenous administration of antibiotics for 6 weeks [11].

\section{Brooker's classification}

\subsection{Introduction}

This system was proposed by Brooker et al. from John Hopkins Hospital in 1973 on a series of 100 consecutive patients undergoing Total hip arthroplasty. Since then it has been in widespread use and has stood the test of time [12]. 


\subsection{Classification}

Class I: Isolated islands of bone.

Class II: Gap between bones at least $1 \mathrm{~cm}$.

Class III: Gap between bones less than $1 \mathrm{~cm}$.

Class IV: Apparent ankylosis.

\subsection{Clinical application}

This classification is useful in the follow up of the high-risk patients and in patients with post $\mathrm{HO}$ resection.

\subsection{Reliability}

Vasileiadis et al. from mayo clinic in their study noted a moderate to substantial agreement (k value $0.49-0.71)$ in the inter observer reliability. Grade IV had best inter observer reliability $[13,14]$ (Figure 8).
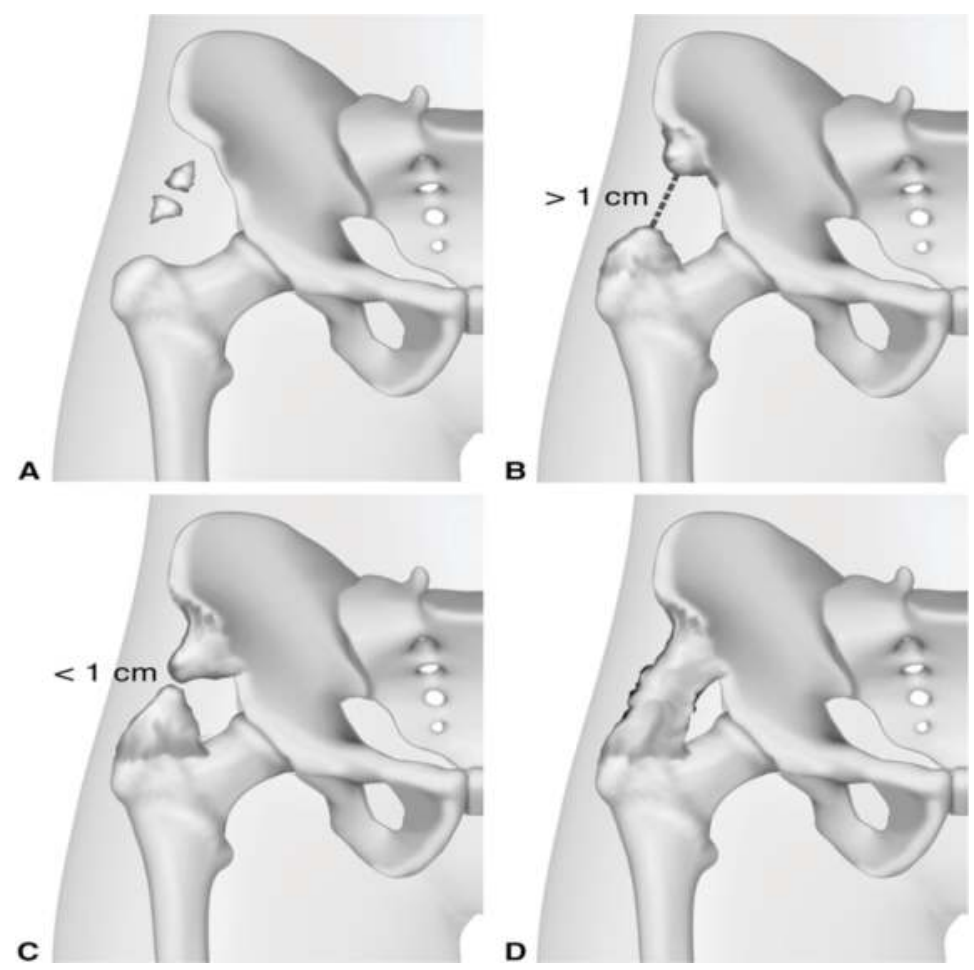

Figure 8. Brooker classification. 


\section{Barrack grading of cementing}

\subsection{Introduction}

This classification was proposed by Barrack et al. based-on review of 50 second generation cemented femoral stems [15].

\subsection{Classification}

Grade A: White-out with complete filling.

Grade B: Slight defects at the cement-bone interface.

Grade C: Defective cement mantle or radiolucency involving 50 to $99 \%$ of the cement-bone interface.

Grade D: $100 \%$ lucency or failure to cover the tip of the stem.

\subsection{Clinical applications}

This classification helps in predicting the survivability of the implant based on the grade of cementing.

\section{Author details}

Munis Ashraf

Address all correspondence to: munis6@gmail.com

KG Hospital, Coimbatore, India

\section{References}

[1] Paprosky WG, Perona PG, Lawrence JM. Acetabular defect classification and surgical reconstruction in revision arthroplasty. A 6-year follow-up evaluation. The Journal of Arthroplasty. 1994 Feb;9(1):33-44

[2] Gozzard C, Blom A, Taylor A, Smith E, Learmonth I. A comparison of the reliability and validity of bone stock loss classification systems used for revision hip surgery. The Journal of Arthroplasty. 2003 Aug;18(5):638-642

[3] Saleh KJ, Holtzman J, Gafni A, Saleh L, Jaroszynski G, Wong P, Woodgate I, Davis A, Gross AE. Development, test reliability and validation of a classification for revision hip arthroplasty. Journal of Orthopaedic Research. 2001 Jan 1;19(1):50-56 
[4] Hodgkinson JP, Shelley P, Wroblewski BM. The correlation between the roentgenographic appearance and operative findings at the bone-cement junction of the socket in Charnley low friction arthroplasties. Clinical Orthopaedics and Related Research. 1988 Mar;228: 105-109

[5] Valle CJ, Paprosky WG. Classification and an algorithmic approach to the reconstruction of femoral deficiency in revision total hip arthroplasty. The Journal of Bone and Joint Surgery. American Volume. 2003;85-A(Suppl 4):1-6

[6] D'Antonio J, McCarthy JC, Bargar WL, Borden LS, Cappelo WN, Collis DK, Steinberg ME, Wedge JH. Classification of femoral abnormalities in total hip arthroplasty. Clinical Orthopaedics and Related Research. 1993 Nov;296:133-139

[7] Dossick PH, Dorr LD, Gruen T, Saberi MT. Techniques for preoperative planning and postoperative evaluation of noncemented hip arthroplasty. Techniques in orthopaedics. 1991 Sep 1;6(3):1-6

[8] Greidanus NV, Mitchell PA, Masri BA, Garbuz DS, Duncan CP. Principles of management and results of treating the fractured femur during and after total hip arthroplasty. Instructional Course Lectures. 2003;52:309-322

[9] Brady OH, Garbuz DS, Masri BA, Duncan CP. The reliability and validity of the Vancouver classification of femoral fractures after hip replacement. The Journal of Arthroplasty. 2000 Jan;15(1):59-62

[10] Rayan F, Dodd M, Haddad FS. European validation of the Vancouver classification of periprosthetic proximal femoral fractures. Journal of Bone and Joint Surgery. British Volume (London). 2008 Dec;90(12):1576-1579

[11] Tsukayama DT, Estrada R, Gustilo RB. Infection after total hip arthroplasty. A study of one hundred and six infections. The Journal of Bone and Joint Surgery. American Volume. 1996;78:512-523

[12] Brooker AF, Bowerman JW, Robinson RA, Riley LH Jr. Ectopic ossification following total hip replacement. Incidence and a method of classification. The Journal of Bone and Joint Surgery. American Volume. 1973 Dec;55(8):1629-1632

[13] Toom A, Fischer K, Märtson A, Rips L, Haviko T. Inter-observer reliability in the assessment of heterotopic ossification: Proposal of a combined classification. International Orthopaedics. 2005 Jun;29(3):156-159

[14] Vasileiadis GI, Itoigawa Y, Amanatullah DF, Pulido-Sierra L, Crenshaw JR, Huyber C, Taunton MJ, Kaufman KR. Intraobserver reliability and interobserver agreement in radiographic classification of heterotopic ossification. Orthopedics. 2017 Jan 24;40(1):e54-e58

[15] Barrack RL, Mulroy RD Jr, Harris WH. Improved cementing techniques and femoral component loosening in young patients with hip arthroplasty. A 12-year radiographic review. Journal of Bone and Joint Surgery. British Volume (London). 1992 May;74(3):38 
